\title{
Electric-field-induced charge-density variations in covalently bonded binary compounds
}

\author{
J. Stahn and U. Pietsch* \\ Institute of Physics, University of Potsdam, Germany
}

P. Blaha and K. Schwarz

Institute of Physical and Theoretical Chemistry, Technical University of Wien, Austria

(Received 26 June 2000; revised manuscript received 10 November 2000; published 4 April 2001)

\begin{abstract}
Extended data sets for the relative intensity variation of x-ray Bragg reflections of GaAs and ZnSe caused by an external electric field were collected using a modulation demodulation technique. In particular, the functional dependence of the intensity variation on field strength and wavelength of the synchrotron radiation was determined. The wavelength dependence allows to determine the phases of the difference-structure factors. An interpretation of all data using a semiempirical model leads to the following conclusions: The main contribution to the measured effects comes from a displacement of the anion sublattice relative to the cation sublattice. The response of the electron density to an external electric field is negligibly small according to density functional theory calculations with both, clamped and "free" ions. This effect is much smaller than the redistribution of the electron density in the bond region associated with the displacements of the atoms causing a change in overlap. The dependence of the sublattice displacement on the strength of the external electric field $\mathbf{E} \|[111]$ is estimated to be $1.5 \times 10^{-8} \AA / \mathrm{V} \mathrm{mm}^{-1}$ for $\mathrm{GaAs}$ and $13.5 \times 10^{-8} \AA / \mathrm{V} \mathrm{mm}{ }^{-1}$ for $\mathrm{ZnSe}$. For the mainly covalent GaAs the inverse piezoelectric effect can be explained by this bond-length variation, in contrast to the much more ionic compound $\mathrm{ZnSe}$ for which this is not the case.
\end{abstract}

DOI: 10.1103/PhysRevB.63.165205

PACS number(s): 77.65.-j, 71.55.Eq, 71.55.Gs, 71.15.Mb

\section{INTRODUCTION}

On the macroscopic scale, the spatial and electronic responses of crystalline semiconductors and insulators to an external electric field are well known as the inverse piezoelectric effect and dielectric polarization, respectively. Both physical properties are used in many technical applications, e.g., piezo speakers, high precision translation stages, or dielectrics for capacitors.

On a microscopic scale, the inverse piezoelectric effect is connected to a redistribution of charged particles or a reorientation of multipolar units leading to a unit-cell distortion. The dielectricity is often explained by a "polarization density" - which is a misleading term for a rigorous description in terms of a charge density. As Martin and Ortiz stated: "The charge density of the electrons is a continuous function of position and there is no way to uniquely cut the density and derive the dipole moment of a cell." 1 An alternative description is the electric current induced in the crystal by an altering external electric field, which is a well-defined quantity. This current is proportional to the time derivative of the polarization. ${ }^{1,2}$

For a quantum-mechanical description of "polarization in a crystal" a geometrical Berry's phase approach has been introduced in recent years. ${ }^{1}$ Unfortunately, this approach is only valid if there is no external electric field in the crystal, which means that the surfaces of the crystal are short circuited. ${ }^{3}$ A general problem in calculating crystal properties in an external electric field is that the total potential $V$ $=V_{\text {int }}+V_{\text {ext }}$ in the Hamiltonian

$$
-\frac{\hbar^{2}}{2 m_{e}} \nabla^{2} \Psi+\left(V_{\mathrm{int}}+V_{\mathrm{ext}}\right) \Psi=E \Psi
$$

is no longer periodic. $V_{\text {int }}$ is the periodic potential caused by all charged particles within the crystal, while $V_{\text {ext }}$ is the external potential from external charges (outside the crystal). The translational symmetry of the wave function $\Psi$ is broken and from this point of view the solid is no longer an ideal crystal. A locally homogeneous external electric field may be simulated by introducing a potential with a period several times the lattice parameters of the crystal. ${ }^{4,5}$ With this super cell method Resta and Baldereschi calculated the electrondensity response in GaAs with a pseudopotential approach and clamped ions. ${ }^{6}$ Up to now there exists no quantummechanical approach to this problem. ${ }^{3}$ Nevertheless, the electron density in a homogeneous external electric field $\rho_{E}$ $=\left|\Psi_{E}\right|^{2}$ is still periodic in three dimensions, but is different from the unperturbed density $\rho_{0}$. The periodic electron density and its change due to an external perturbation can be measured by $x$-ray scattering. This provides an opportunity to check ab initio or semiempirical approaches to describe the charge-density response to an external electric field.

The $\mathrm{x}$-ray probe is indirect because there is no unambiguous way to extract the electron-density $\rho$ from a limited set of measured integral intensities $\{R\}$ of Bragg reflections. This is shown by

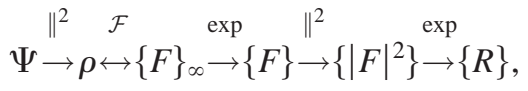

where "exp" stands for experimental conditions and influences: limited data sets, temperature effects, anomalous dispersion, and the like, \{\}$_{\infty}$ means unlimited data set. Except for the Fourier transform $\mathcal{F}$ all steps are not reversible, in particular, the phases of the complex structure factors $F$ are lost since $R$ is proportional to $|F|^{2}$. 


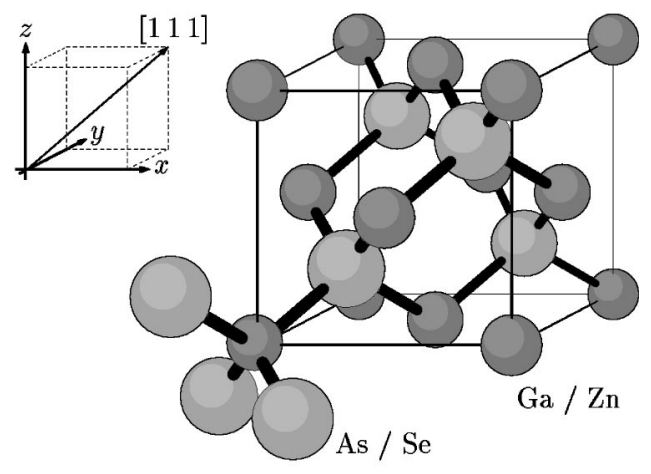

FIG. 1. Unit cell of the zinc-blende crystal structure. The origin is located at cation site. The black lines symbolize covalent bonds. $1 / 4$ of these bonds is directed parallel to [111].

In x-ray structure analysis the measured intensity variations caused by the external electric field have to be explained in terms of a charge-density model. A simple but instructive model is the superposition of spherical atoms $i$ at location $\mathbf{r}_{i}$ that leads to the structure factor

$$
F(\mathbf{k})=\sum_{i} f_{i}(k) \exp \left[i \mathbf{k} \mathbf{r}_{i}\right]
$$

with the atomic form factors $f_{i}(k)$ and where $\mathbf{k}$ is the scattering vector.

The isoelectronic compounds GaAs and $\mathrm{ZnSe}$ form isolating crystals of the zinc blende type. All cations are tetrahedrally coordinated by anions and vice versa (see Fig. 1). The unit cell is of cubic symmetry but there is no center of inversion that would prevent the occurence of small changes in the electron density. This, and the good reliability of large crystals, makes them ideal subjects to study the mentioned effects.

The zinc blende structure provides three types of structure factor: For $h+k+l=4 n+2$ (weak reflections) the phase relation between the anion and cation sublattices is about -1 , which means that the core contributions to the structure factor almost cancel [see Eq. (3) and Eq. (5)]. For similar atomic numbers of cation and anion (as in GaAs and $\mathrm{ZnSe}$ ) the resulting structure factor amplitude is about two orders of magnitude smaller than for the strong $(h+k+l=4 n)$ and medium intensity $(h+k+l=4 n \pm 1)$ reflections. The weak reflections are much more sensitive to changes in the electron density of a crystal. ${ }^{7}$ The rather diffuse charge density in the bonding region and the charge transfer between cation and anion are mainly associated with the low-indexed reflections ( $\sin \theta / \lambda<0.5 \AA$ ) whereas a shift in the atomic positions is associated with the higher-order reflections via the phase factor $\exp [i \mathbf{k r}]$. Thus, the measurement of both high- and low-indexed reflections gives the opportunity to distinguish between the possible model explanations for the inverse piezoelectric effect on the electron-density level.

The weak reflections 222 and $\overline{2} \overline{2} \overline{2}$ are the ones that are most sensitive to a rearrangement of the electron density in the bond region in the [111] direction. Thus, they are taken as representatives for all weak reflections in the following discussion.
A change in the electron-density distribution caused by the external electric field results in a variation of the modulus and the phases of the x-ray structure factors and in changed lattice parameters. The latter effect is known as the inverse piezoelectric effect and is expressed by

$$
\mathbf{r}^{\prime}=(1+\boldsymbol{\epsilon}) \mathbf{r}, \quad \boldsymbol{\epsilon}=\mathbf{d E}
$$

where $\mathbf{r}^{\prime}$ and $\mathbf{r}$ are the lattice vectors, $\mathbf{E}$ is the electric field vector, $\boldsymbol{\epsilon}$ is called strain tensor, $\mathbf{d}$ is the piezoelectric tensor, ${ }^{8}$ which for zinc-blende-type crystals contains only one independent nonzero coefficient $d_{123}=d_{132}=\ldots$ (and all permutations).

For an external electric field $\mathbf{E} \|[111]$ with $E$ $=1 \mathrm{kV} \mathrm{mm}^{-1}$ the relative length variation of the diagonal of the unit cell in the direction of the field is $2 \epsilon$ $=2 d_{123, \mathrm{GaAs}} E / \sqrt{3}=3.1 \times 10^{-6}$ for GaAs and $1.3 \times 10^{-6}$ for $\mathrm{ZnSe}$. These small values suggest that the inverse piezoelectric effect hardly affects the position and intensity of the Bragg reflections. Indeed, a change in the direction of the reflected beam larger than the experimental angular resolution of $0.001^{\circ}$ could only be observed for reflections with $\sin \theta / \lambda \geqslant 0.9 \AA$.

Since both, the piezoelectric coefficients and the respective shift of the Bragg angle are related to lattice parameters only, there is no information about the change in the relative coordinates of atoms and the respective charge-density response within the unit cell. For the piezoelectric effect (creation of an electric field in the crystal by the application of external pressure) the "internal strain effect" as a result of an uniaxial stress along [111] has already been measured. ${ }^{9-14}$ In this case, the anion sublattice does not completely follow the cation sublattice upon compression or expansion as expected for a homogeneously deformed system. This means, that the bond lengths remain almost fixed but the unit-cell distortion is mainly associated with a change in the bond angles.

Earlier experiments probing the charge-density response in GaAs to an external electric field by Fujimoto ${ }^{15}$ and by Pietsch, Mahlberg, and Unger ${ }^{16}$ were limited by having no synchrotron radiation source available. At that time only a few reflections could be measured at one wavelength due to the low intensity of the X-ray tubes. These very limited data sets were interpreted by Fujimoto in terms of ionicity and internal strain and by Pietsch, Mahlberg, and Unger as bond charge rearrangement.

To check these first interpretations and to provide an experimental basis for the discussion of theoretical concepts of screening in insulators, these measurements were repeated and extended to include more reflections, various field strengths, wavelengths, and to the more ionic compound $\mathrm{ZnSe}$ (Sec. II). In Sec. III and Sec. IV the experimental findings are interpreted semiempirically as a displacement of the cation and anion sublattices relative to each other. This interpretation was cross-checked by performing density functional theory (DFT) calculations (Sec. V). 


\section{EXPERIMENTS}

Special attention was directed to the determination of the electrical-field induced variation of the integrated intensities $R$ of Bragg reflections. $R$ was determined by measuring rocking curves around the exact Bragg position. Since the relative intensity variation $(\Delta R / R)(E, \lambda)$ due to the external electric field is of the order of $1 \%$ or less, a modulation demodulation technique was used. ${ }^{13,15}$ This means that for each point on the rocking curve the external field was switched on and off 100 times with a frequency of about 20 $\mathrm{Hz}$. After (dis-) chargement of the plate capacitorlike sample the detected signal was collected in different counters for each external voltage. ${ }^{17}$ By this method, the intensities with $\left(R_{E}\right)$ and without $\left(R_{0}\right)$ an electric field were measured quasisimultaneously and, therefore, the relative intensity variation is not affected by variations in the synchrotron radiation characteristics (such as intensity, polarization, or beam position) on a time scale larger than $1 \mathrm{~s}$.

The rocking curves were scanned with about 80 positions, each of $5 \mathrm{~s}$ measuring time. To get sufficient statistical accuracy, this procedure was repeated up to 40 times with identical experimental parameters.

The samples were single-crystalline wafers of 0.5 to 1 $\mathrm{mm}$ thickness and 2 to $5 \mathrm{~cm}^{2}$ area, which were prepared as plate capacitors by evaporating silver spots as electrodes on both surfaces. The voltage was applied to these spots via copper wires.

All measurements were performed at the synchrotron radiation source HASYLAB at beamline D3. A detailed description of the experimental setup and of the modulation demodulation technique is given elsewhere. ${ }^{17,18}$ There, part of the data used here was already published.

A reduction of the measured intensity to structure factors $F$ requires model assumptions and the knowledge of the structure factor phases. Therefore, the subsequent discussion will be based on the relative intensity variation $(\Delta R / R)$ $\approx\left(\left|F_{E}\right|^{2}-\left|F_{0}\right|^{2}\right) /\left|F_{0}\right|^{2}$ where experimental corrections in $\Delta R$ compensate for each other. The assumption $R \propto|F|^{2}$ is based on the kinematic theory of $\mathrm{x}$-ray scattering, which is a good approximation for weak reflections. The other reflections may be affected by extinction, which in turn may depend upon the external field strength. In this case a discussion of the induced intensity variations is much more complicated.

The following experimental parameters were varied: (i) direction of the external electric field, (ii) the type of reflection, and (iii) the wavelength of the synchrotron radiation.

(i) The available electric field strength for GaAs was limited to $E<3.5 \mathrm{kV} \mathrm{mm}^{-1}$ by a possible electrical break down of the sample under simultaneous irradiation. Measurements were performed with the external electric field parallel to one of the four chemical bonds connecting each atom to its nearest neighbors ([111] direction) and parallel to [100].

The relative intensity variation of 17 weak reflections of $\mathrm{GaAs}$ and $\mathrm{ZnSe}$ were measured for several field strengths. The maximum value for $(\Delta R / R)$ is about $2 \%$ for GaAs and $3 \%$ for $\mathrm{ZnSe}$. For all reflections, an almost linear dependence of $(\Delta R / R)$ on $E$ was found for one direction of $\mathbf{E}$. But for
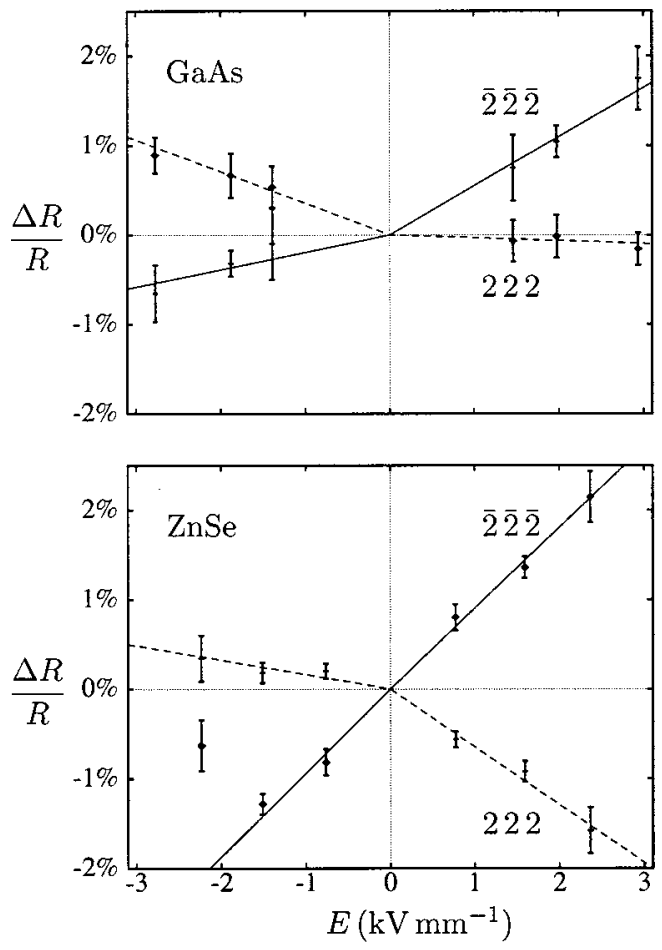

FIG. 2. Relative intensity variation $\Delta R / R$ for the reflections 222 and $\overline{2} \overline{2} \overline{2}$ of GaAs (top) and $\mathrm{ZnSe}$ (bottom) as functions of the field strength. The wavelength is $0.99 \AA$ for GaAs and $0.96 \AA$ for $\mathrm{ZnSe}$. The field is parallel to [111]. The lines of regression show different slopes for opposite field directions, which is a typical feature for all cases with $\mathbf{E} \|[111]$.

$\mathbf{E} \|[111]$ the slope of the line of regression was different for opposite field directions as demonstrated for the examples 222 and $\overline{2} \overline{2} \overline{2}$ in Fig. 2. The strength of the effect is of the same order of magnitude for all weak reflections-even for the rather high-indexed 666. This means that the measured effect is not only due to a change in the bond charges but also the charge densities close to the nuclei are affected.

Table I shows the slopes of the lines of regression $(\Delta R / R)(E)$ for a series of reflections of GaAs together with the corresponding results of the semiempirical bond-charge model (Sec. IV) and of the DFT calculations (Sec. V). The different slopes for $E>0$ and for $E<0$ may be due to the influence of space charges close to one of the electrodes (see later). The differences among the reflections $h k l$ and $\bar{h} \bar{k} \bar{l}$ are caused by the effect of anomalous dispersion close to the absorption edges, which breaks Friedel's law. For E $\|[100]$ the lines of regression for the two field directions coincide.

(ii) For the measured strong and medium $\mathrm{ZnSe}$ reflections $\{111\},\{333\}$, and $\{444\}$ the relative intensity variation is smaller than the experimental error of $0.05 \%$. This means that for these reflections, the effect is at least 2 orders of magnitude smaller than for the weak reflections. This is to be expected, since the absolute changes in $F$ induced by the electric field are estimated to be about the same for all reflections with similar $\sin \theta / \lambda$.

(iii) As will be discussed later, the difference-structure factor $\Delta F$ describing the change in the electron density is 
TABLE I. Slopes of the lines of regression for $(\Delta R / R)(E)$ obtained for the experimental values of GaAs $(\mathbf{E} \|[111])$. The slopes for $E>0$ and $E<0$ were determined separately. Columns 5 and 6 give the slopes obtained with the semiempirical model and by DFT calculations, respectively. All values are given in $\% / \mathrm{kV} \mathrm{mm}^{-1}$.

\begin{tabular}{|c|c|c|c|c|c|}
\hline \multirow[b]{2}{*}{$\lambda(\AA)$} & \multirow[b]{2}{*}{$h k l$} & \multicolumn{2}{|c|}{ Experiment } & \multirow[b]{2}{*}{ Model } & \multirow[b]{2}{*}{ DFT } \\
\hline & & $E<0$ & $E>0$ & & \\
\hline \multirow[t]{2}{*}{0.70} & $\overline{2} \overline{2} \overline{2}$ & $0.00(11)$ & $-0.01(11)$ & -0.02 & 0.02 \\
\hline & $\overline{6} \overline{6} \overline{6}$ & $-0.05(10)$ & $-0.27(9)$ & 0.06 & 0.07 \\
\hline \multirow[t]{2}{*}{0.80} & $\overline{2} \overline{2} \overline{2}$ & $-0.01(37)$ & $0.01(35)$ & -0.00 & 0.04 \\
\hline & $\overline{6} \overline{6} \overline{6}$ & $-0.12(25)$ & $0.38(25)$ & 0.08 & 0.10 \\
\hline \multirow[t]{3}{*}{0.90} & 222 & $-0.16(3)$ & $0.02(11)$ & -0.21 & -0.14 \\
\hline & $\overline{2} \overline{2} \overline{2}$ & $0.09(4)$ & $0.05(4)$ & 0.07 & 0.09 \\
\hline & $\overline{6} \overline{6} \overline{6}$ & $0.13(30)$ & $0.38(30)$ & 0.14 & 0.18 \\
\hline \multirow[t]{3}{*}{0.95} & 222 & $-0.30(1)$ & $0.01(6)$ & -0.28 & -0.20 \\
\hline & $\overline{2} \overline{2} \overline{2}$ & $0.16(19)$ & $0.11(18)$ & 0.22 & 0.21 \\
\hline & 666 & $-0.45(14)$ & $0.11(12)$ & -0.20 & -0.26 \\
\hline \multirow[t]{3}{*}{0.97} & 222 & $-0.32(8)$ & $0.05(7)$ & -0.31 & -0.23 \\
\hline & $\overline{2} \overline{2} \overline{2}$ & $0.12(2)$ & $0.38(11)$ & 0.38 & 0.35 \\
\hline & $\overline{4} \overline{4} \overline{2}$ & $0.07(5)$ & $0.27(6)$ & 0.21 & 0.20 \\
\hline \multirow[t]{17}{*}{0.99} & 200 & $-0.02(15)$ & $-0.14(15)$ & -0.42 & -0.14 \\
\hline & $\overline{2} 00$ & $0.12(9)$ & $0.12(8)$ & 0.42 & 0.14 \\
\hline & 222 & $-0.35(2)$ & $-0.04(1)$ & -0.32 & -0.25 \\
\hline & $\overline{2} \overline{2} \overline{2}$ & $0.17(3)$ & $0.54(2)$ & 0.54 & 0.59 \\
\hline & $\overline{2} 22$ & $-0.15(21)$ & $-0.07(20)$ & -0.18 & -0.20 \\
\hline & $2 \overline{2} \overline{2}$ & $0.09(2)$ & $0.10(3)$ & 0.11 & 0.09 \\
\hline & 420 & $-0.43(2)$ & $0.09(4)$ & -0.21 & -0.28 \\
\hline & $\overline{4} \overline{2} 0$ & $-0.14(11)$ & $0.67(33)$ & 0.19 & 0.28 \\
\hline & 442 & $-0.39(2)$ & $0.02(2)$ & -0.25 & -0.28 \\
\hline & $\overline{4} \overline{4} \overline{2}$ & $0.06(10)$ & $0.35(5)$ & 0.31 & 0.29 \\
\hline & $\overline{6} 00$ & $0.10(10)$ & $0.08(10)$ & 0.12 & 0.17 \\
\hline & 622 & $-0.17(16)$ & $-0.01(16)$ & -0.20 & -0.25 \\
\hline & $\overline{6} \overline{2} \overline{2}$ & $0.14(6)$ & $0.24(15)$ & 0.24 & 0.25 \\
\hline & 640 & $-0.46(24)$ & $0.09(22)$ & -0.21 & -0.23 \\
\hline & $\overline{6} \overline{4} 0$ & $0.02(16)$ & $0.34(16)$ & 0.22 & 0.24 \\
\hline & 666 & $-0.77(15)$ & $-0.01(12)$ & -0.36 & -0.32 \\
\hline & $\overline{6} \overline{6} \overline{6}$ & $0.00(1)$ & $0.61(5)$ & 0.31 & 0.35 \\
\hline \multirow[t]{3}{*}{1.00} & 222 & $-0.27(1)$ & $-0.07(1)$ & -0.30 & -0.25 \\
\hline & $\overline{2} \overline{2} \overline{2}$ & $0.34(7)$ & $0.40(5)$ & 0.52 & 0.58 \\
\hline & $\overline{6} \overline{6} \overline{6}$ & $0.06(6)$ & $0.38(7)$ & 0.31 & 0.34 \\
\hline 1.01 & $\overline{2} \overline{2} \overline{2}$ & $0.18(6)$ & $0.44(7)$ & 0.40 & 0.40 \\
\hline 1.10 & $\overline{2} \overline{2} \overline{2}$ & $-0.09(4)$ & $0.01(2)$ & -0.09 & -0.08 \\
\hline
\end{tabular}

" 'amplified" by the wavelength dependent-structure factor $F$. This "amplification" has its maximum close to the $K$-absorption edge of As and Se, respectively. Therefore, the wavelength of the synchrotron radiation $\lambda$ was varied in the range 0.7 to $1.1 \AA$. The corresponding dependence of $(\Delta R / R)_{22} \overline{2}$ upon $\lambda$ is shown in Fig. 3 for both substances. $(\Delta R / R)_{E}(\lambda)$ shows a distinct extremum close to the $K$ absorption edge of the respective anion. This behavior is caused by the wavelength dependence of anomalous disper-
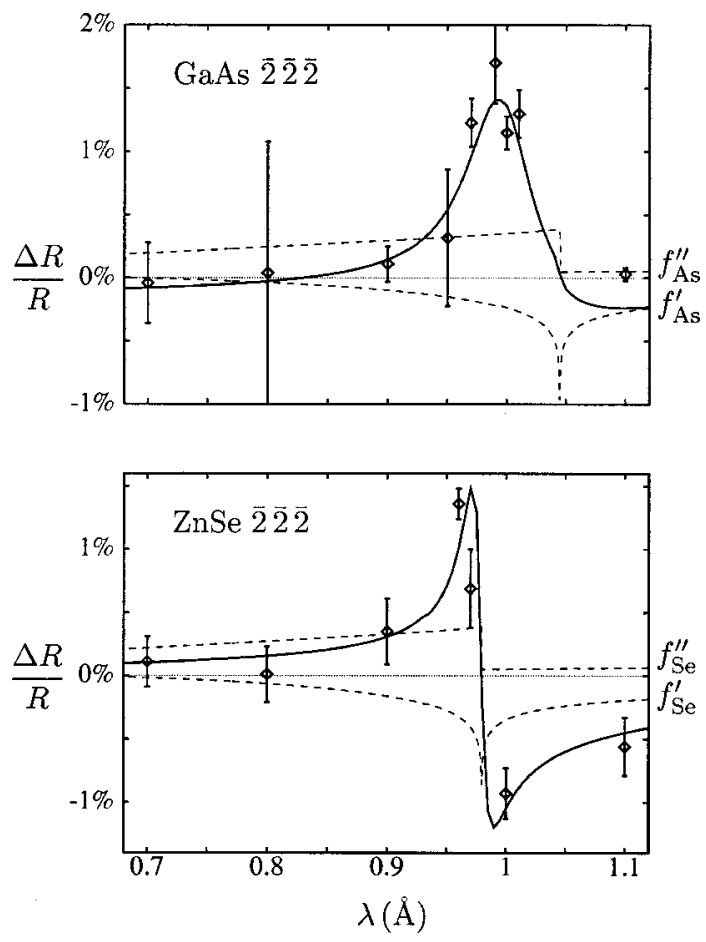

FIG. 3. Wavelength dependence of $(\Delta R / R)_{222}^{-\overline{2}}$ of GaAs (top) and $\mathrm{ZnSe}$ (bottom). The solid lines are simulated curves as described in Sec. IV. The dotted lines show the functional behavior of the real and the imaginary part of the anomalous dispersion connected to As and Se, respectively.

sion and allows to extract phase information, as will be discussed in the next section.

\section{PHASE INFORMATION}

In a simple picture, the electron density of a crystal $\rho(\mathbf{r})$ can be approximated by a superposition of (charged) spherical atoms and "bond charges", representing the charge accumulation between nearest neighbors due to a covalent bond. ${ }^{17,30,31}$ This ansatz leads to the expression

$$
\begin{aligned}
F(\mathbf{k})= & 4\left(f_{c}+f_{c}^{\prime}+i f_{c}^{\prime \prime}\right) T_{c}+4\left(f_{a}+f_{a}^{\prime}+i f_{a}^{\prime \prime}\right) T_{a} e^{i \mathbf{k} \mathbf{r}_{a}} \\
& +4 \sum f_{b c} e^{i \mathbf{k r}_{b c}},
\end{aligned}
$$

where $f_{c, a}$ are the atomic form factors of the cation or anion, respectively; $f_{c, a}^{\prime}+i f_{c, a}^{\prime \prime}$ are the corresponding coefficients of the anomalous dispersion and $T_{c, a}$ are the Debye-Waller factors (see Sec. IV). $f_{b c}$ is the bond-charge form factor. In the following, $F$ will be split in its real $(\operatorname{Re}[F])$ and imaginary part $(\operatorname{Im}[F])$.

The strong dependence of $(\Delta R / R)$ upon $\lambda$ (see Fig. 3) can be explained by the influence of anomalous dispersion. The structure factor of the perturbed crystal can be written as $F$ $=F_{0}+\Delta F$, where $\Delta F$ is the change caused by the external perturbation. This leads to: 

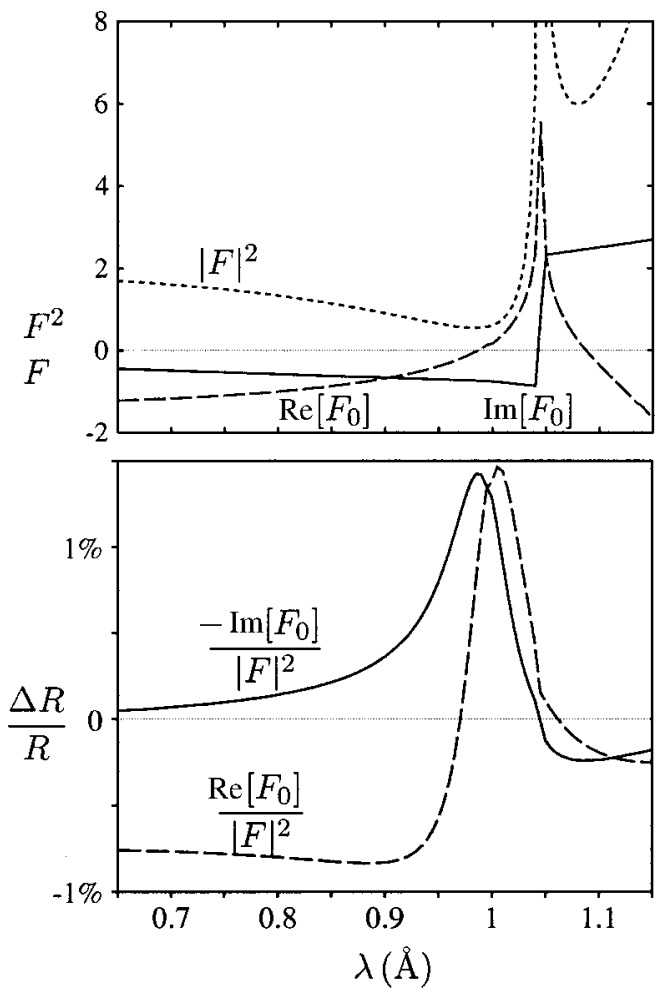

FIG. 4. Wavelength dependence of contributions to Eq. (7) for the reflection $\overline{2} \overline{2} \overline{2}$ for GaAs (top) and the resulting contributions to $\Delta R / R$, scaled to the order of the experimental results (bottom).

$$
\begin{aligned}
\left|F_{E}\right|^{2} & -\left|F_{0}\right|^{2} \\
& =\operatorname{Re}^{2}\left[F_{0}+\Delta F\right] \operatorname{Im}^{2}\left[F_{0}+\Delta F\right]-\operatorname{Re}^{2}\left[F_{0}\right]-\operatorname{Im}\left[F_{0}\right] \\
& \approx 2 \operatorname{Re}\left[F_{0}\right] \operatorname{Re}[\Delta F]+2 \operatorname{Im}\left[F_{0}\right] \operatorname{Im}[\Delta F]+O^{2} .
\end{aligned}
$$

The wavelength dependence of $f_{c, a}^{\prime}+i f_{c, a}^{\prime \prime}$ (see Fig. 3) leads to different wavelength dependences in $\operatorname{Re}\left[F_{0}\right]$ and $\operatorname{Im}\left[F_{0}\right]$. Figure 4 shows these quantities calculated for GaAs without bond charges together with $\left|F_{0}\right|^{2} .\left|F_{0}\right|^{2}$ has a minimum at about $1 \AA$ caused by $\operatorname{Re}\left[F_{0}\right] \approx 0$. This minimum is responsible for the maximum in $(\Delta R / R)$ in Fig. 3. A comparison of the wavelength dependences of $\operatorname{Re}\left[F_{0}\right] /\left|F_{0}\right|^{2}$ and $\operatorname{Im}\left[F_{0}\right] /\left|F_{0}\right|^{2}$ shown in Fig. 4 with the experimentally determined curves in Fig. 3 shows a good agreement in shape, asymptotic behavior, and zero points with $\operatorname{Im}\left[F_{0}\right]\left|F_{0}\right|^{2}$. This means that $\operatorname{Re}[\Delta F] \approx 0$ and that the phase of $\Delta F$ is about $\pm \pi / 2$.

Calculations including bond charges and Debye-Waller factors show that this phase may be explained by the displacement of the anion relative to the cation sublattice. For weak reflections the phase factor of the anion is $\exp \left[i \mathbf{i} \mathbf{r}_{a}\right]$ $\approx-1+i \mathbf{k} \mathbf{u}_{a},\left(\mathbf{r}_{c}:=0\right)$, where $\mathbf{u}_{a}$ is the displacement of the anion from the position in the unperturbed state. Any change in the bond-charge parameters $\mathbf{r}_{b c}$ and $f_{b c}$ or in the extent of $f_{c, a}$ essentially leads to a phase for $\Delta F$ of 0 or $\pi$, which means that they hardly contribute to the measured effect.

In Fig. 5, the quantities $\Delta \varphi, \varphi(\Delta F), F_{E}$, and $F_{0}$ are shown for the GaAs reflection 222. A more detailed analysis of the measured $(\Delta R / R)$ (see next section) predicts $\Delta \varphi$ $\approx 0.3^{\circ}$. An attempt to measure directly the phase shift via

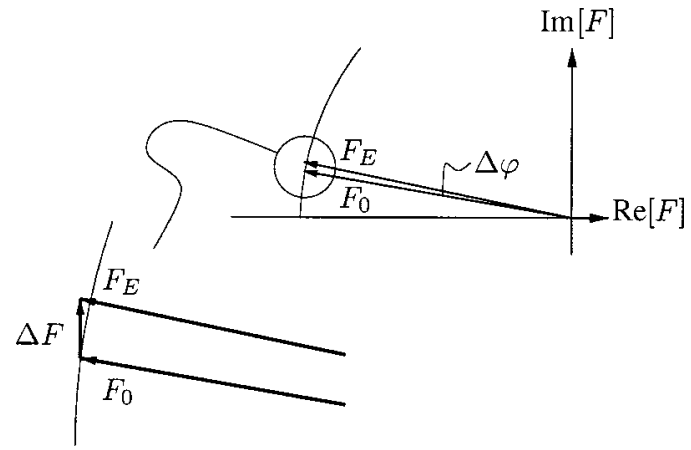

FIG. 5. Roughly scaled sketch of the complex structure factors $F_{0}$ and $F_{E}$ for the GaAs reflection 222 without anomalous dispersion and temperature effects. The phase of $F_{0}$ is about $170^{\circ} . \Delta \varphi$ is the phase difference that was estimated to be of the order of $1^{\circ}$ with the three beam case. The difference-structure factor $\Delta F$ has a phase of about $90^{\circ}$ as deduced from the wavelength dependence of $(\Delta R / R)$.

triple-beam interferences ${ }^{32,33}$ supports this result qualitatively. There the phase variation was determined to be of the same sign and of the order of $1^{\circ} .{ }^{18,34}$

\section{SEMIEMPIRICAL MODEL}

From the considerations in Sec. III it follows that the difference-structure factor has the phase factor $\pm \pi / 2$, or in other words, that it is mainly the imaginary part of the structure factor $F$ that is altered by the external electric field. In Eq. (5) both the anion position, via the phase factor $\exp \left[i \mathbf{k r}_{a}\right]$, and the imaginary part of the Debye-Waller factor $T_{i}$ contribute to the scattering phase of the anion relative to the cation. The imaginary part of $T_{i}$ is connected to the anharmonicity of the vibrating potential of the ions-and thus to the mean ionic position.

According to Willis, ${ }^{35}$ the effect of thermal vibration of an "atom" in a cubic crystal upon the structure factor can be estimated by assuming an independent motion of the individual ions in an anharmonic internal potential $V_{\text {int }}(\mathbf{u})$ caused by the surrounding ions.

$$
V_{\text {int }}(\mathbf{u})=V_{0}+\frac{1}{2} \alpha u^{2}+\beta u_{1} u_{2} u_{3}+O^{4},
$$

where $\mathbf{u}$ is the displacement from the mean position, $\alpha$ is the harmonic force constant, and $\beta$ is the tetrahedral anharmonic constant. As a result, the equilibrium site of the nucleus $\mathbf{r}$ is then convoluted with

$$
T_{i}(\mathbf{u})=\frac{\exp \left[\frac{-V_{i}\left(T, \mathbf{u}_{i}\right)}{k_{B} T}\right]}{\int_{-\infty}^{\infty} \exp \left[\frac{-V_{i}\left(T, \mathbf{u}_{i}\right)}{k_{B} T}\right] d \mathbf{u}_{i}} .
$$

In reciprocal space, this means a multiplication of the atomic form factors with the Debye-Waller factor $T_{i}(\mathbf{k})$, which is the complex Fourier transform of $T_{i}(\mathbf{u})$.

If the regarded "atom" is charged, there will be an additional contribution $V_{\text {ext }}(\mathbf{E}, \mathbf{u})=\mathbf{E} \mathbf{u} \gamma$ to the potential, where 
$\gamma_{i}$ is the effective charge of the ion: $V(\mathbf{E}, \mathbf{u})=V_{\text {int }}(\mathbf{u})$ $+V_{\text {ext }}(\mathbf{E}, \mathbf{u})$. As a consequence, the anharmonicity is increased or decreased in field direction. This leads to the modified Debye-Waller factor

$$
\begin{aligned}
T_{i}(\mathbf{k})= & \exp \left[-\frac{\sin ^{2} \theta}{\lambda^{2}} B_{i}\right][1+i \underbrace{\frac{\beta_{i} h k l}{k_{B} T}\left(\frac{B_{i}}{4 \pi a_{0}}\right)^{3}}_{\text {thermal }} \\
& +i \underbrace{\frac{\gamma_{i} E(h+k+l)}{\sqrt{3} k_{B} T}\left(\frac{B_{i}}{4 \pi a_{0}}\right)}_{\text {static, caused by } \mathbf{E}}]
\end{aligned}
$$

being used in Eq. (5). $B_{i}=8 \pi^{2} k_{B} T / \alpha_{i}$ is the temperature factor of the atom. Via Eq. (9) and Eq. (5) $\gamma_{c}=-\gamma_{a}$ can be refined so that $(\Delta R / R)_{\text {calc }}=\left(\left|F_{E}\right|^{2}-\left|F_{0}\right|^{2}\right) /\left|F_{0}\right|^{2}$ fits the experimental data.

The experimentally obtained $\lambda$ dependence can be reproduced quantitatively and qualitatively for one field direction at a time. A small discrepancy remains for GaAs $\overline{2} \overline{2} \overline{2}$ and $\lambda \approx 0.9 \AA$. This ansatz leads to an almost linear dependence of $(\Delta R / R)$ on $E$ for $0 \leqslant|E| \leqslant 3 \mathrm{kV} \mathrm{mm}^{-1}$ and is not able to reproduce the different slopes for opposite field directions.

Since cations and anions are displaced in opposite directions, the bond-length parallel to the field becomes stretched (or compressed) and the remaining bonds are compressed (or stretched). This means that the bond angles are distorted. The distortion of the unit cell, which is a consequence of the relaxation of the distorted bond angles, is included in the model by reducing the symmetry and modifying the lattice parameters according to Eq. (4). This inverse piezoelectric effect hardly changes the calculated relative intensity variations because the essential parameters entering Eq. (5) are the relative atomic positions in the unit cell. In other words, a homogeneous distortion of the unit cell changes both $\mathbf{k}$ and $\mathbf{r}_{i}$ so that $\mathbf{k r} \mathbf{r}_{i} \approx$ const.

If, in addition to the presented model explanation, one assumes a redistribution of the bond charge away from the stretched bonds towards the compressed ones, the above mentioned discrepancy in $(\Delta R / R)_{E}(\lambda)$ for GaAs $\overline{2} \overline{2} \overline{2}$ can be reduced. This corresponds to an increasing overlap of the atomic densities in the compressed bond region and vice versa. The change for the bond parallel to $\mathbf{E}$ is about $3 \%$ for $E=1 \mathrm{kV} \mathrm{mm}{ }^{-1}$. Due to the much higher ionicity in $\mathrm{ZnSe}$, there is practically no influence of the bond charge in this compound.

The effective ionic charges were determined to be $\gamma_{\mathrm{Ga}}$ $=-\gamma_{\mathrm{As}}=0.26 \mathrm{e}$ and $\gamma_{\mathrm{Zn}}=-\gamma_{\mathrm{Se}}=1.6 \mathrm{e}$. Using the solid state Hartree-Fock program CRYSTAL95 (Ref. 36) the respective charges were calculated to be 0.208 and $1.857 \mathrm{e}$.

The displacement of the sublattices relative to each other can be determined from thermodynamics using
TABLE II. Property parameters of GaAs and $\mathrm{ZnSe}$ used in this article. $a_{0}$ : lattice parameter $B_{c, a}$ : harmonic temperature factors $\beta_{c}\left(=-\beta_{a}\right)$ : anharmonic temperature factors $d_{123}$ : piezoelectric coefficient $\epsilon_{0}$ : dielectric constant and $\lambda_{c, a}: K$-absorption critical wavelengths. " $c$ " means cation, " $a$ " means anion; all data correspond to room temperature. Data in Refs. 19-23 were taken from Ref. 24

\begin{tabular}{lllll}
\hline \hline & \multicolumn{1}{c}{ GaAs } & Ref. & \multicolumn{1}{c}{ ZeSe } & Ref. \\
\hline$a_{0}$ & $5.65325(2) \AA$ & 19 & $5.6687 \AA$ & 20 \\
$B_{c}$ & $0.666(4) \AA^{2}$ & 25 & $1.020(5) \AA^{2}$ & 26 \\
$B_{a}$ & $0.566(4) \AA^{2}$ & 25 & $0.739(8) \AA^{2}$ & 26 \\
$\beta_{a}$ & $-1.75 \times{ }^{-19} \mathrm{~J}^{-3}$ & 27 & $-5.6(9) \times{ }^{-19} \mathrm{~J}^{-3}$ & 26 \\
$d_{123}$ & $2.7(1) \times{ }^{-9} \mathrm{~mm} \mathrm{~V}^{-1}$ & 21 & $1.10(6) \times{ }^{-9} \mathrm{~mm} \mathrm{~V}^{-1}$ & 22 \\
$\epsilon_{0}$ & $12.91(5)$ & 23 & 9.6 & 28 \\
$\lambda_{c}$ & $1.1960 \AA$ & 29 & $1.2836 \AA$ & 29 \\
$\lambda_{a}$ & $1.0448 \AA$ & 29 & $0.9795 \AA$ & 29 \\
\hline \hline
\end{tabular}

$$
\begin{aligned}
\left\langle\mathbf{u}_{i}\right\rangle & =\frac{\int \exp \left[-V_{i}(T, \mathbf{u}) / k_{B} T\right] \mathbf{u} d \mathbf{u}}{\int \exp \left[-V_{i}(T, \mathbf{u}) / k_{B} T\right] d \mathbf{u}}, \\
& =-\beta_{i} \frac{3 k_{B} T}{\alpha_{i}^{2}}+\gamma_{i} \frac{E}{\alpha_{i}}
\end{aligned}
$$

The first term on the right side describes the thermal expansion of the crystal and is already considered in the roomtemperature lattice parameters. With the temperature factor $B_{i}=8 \pi^{2} k_{B} T / \alpha_{i}$ one gets

$$
\left\langle\mathbf{u}_{a}\right\rangle-\left\langle\mathbf{u}_{c}\right\rangle=\frac{E}{8 \pi^{2} k_{B} T}\left(\gamma_{a} B_{a}-\gamma_{x} B_{c}\right) .
$$

Using $\gamma_{a}=-\gamma_{c}$ and the parameters given in Table II one can determine the bond-length variation to be $1.5 \times 10^{-5} \AA$ for GaAs and $13.5 \times 10^{-5} \AA$ for $\mathrm{ZnSe}$, both for an external field strength of $1 \mathrm{kV} \mathrm{mm}^{-1}$ with $\mathbf{E} \|[111]$.

Since the bonds in GaAs are almost covalent, nearestneighbor interactions are sufficient to describe the distortion of the unit cell. Due to the tetrahedral coordination of each atom, the bond-length variation described above is partially compensated for by bond-angle variations of bonds that are not parallel to [111]. If one assumes that both effects result in about the same relative variation of the length of the diagonal, but with opposite sign, one can estimate the relative elongation of the unit cell in [111] direction to be $\approx 2\left(\left\langle\mathbf{u}_{a}\right\rangle\right.$ $\left.-\left\langle\mathbf{u}_{c}\right\rangle\right) / \sqrt{3} a_{0}=3 \times 10^{-6}$. This is approximately the value obtained from Eq. (4): $3.1 \times 10^{-6}$. Thus, for covalently bonded crystals the bond-length variation, caused by the forces of the external electric field acting on the charged "atoms," directly leads to the distortion of the unit cell and, thus, to the piezoelectric coefficient.

A similar calculation for $\mathrm{ZnSe}$ leads to a length variation of the unit-cell diagonal of about 20 times the macroscopically measured value. The reason is the large effective charge entering Eq. (10). As for ionic compounds, nextnearest-neighbor interactions become more important and the considerations of the previous paragraph are no longer 
valid. In terms of the internal potential [Eq. (7)] this means that the expansion has to include fourth- and higher-order terms.

The solid lines in Fig. 3 and the slopes of $(\Delta R / R)(E)$ for several reflections of GaAs given in the 5th column of Table I were calculated with the mentioned parameters, "effective charge" and "bond charge transfer" and including the piezoelectric effect. The model reproduces sign and amount of the experimental values. Similar agreement has been obtained for $\mathrm{ZnSe}$ (not shown here). The different slopes of $(\Delta R / R)(E)$ for $E>0$ and $E<0$ are not to be explained with the chosen model. The fact that for $\mathbf{E} \|[111]$ or $\mathbf{E} \|[\overline{1} \overline{1} \overline{1}]$ the smaller slope occurs always when the in- and outgoing beam passes the negatively charged electrode suggests that a surface effect plays a role. Indeed, the thickness of the space charge and the penetration depth for the low-indexed reflections are both of the order of $1 \mu \mathrm{m}$. On the other hand, this would not explain the different slopes for high-indexed reflections with larger penetration depths.

The presented model explanation leads to a correction to the form factor of the type $f_{i} \exp \left[i \mathbf{k r}_{i}\right] T_{i}(1+i \ldots)$ for each atom $i$. Similar mathematical expressions can be derived if a polarization of the atomic cores or a displacement of the nuclei is assumed as the origin of the measured effect. In the first case, $f_{i}$ becomes complex, in the latter the phase of $\exp \left[i \mathbf{k r}_{i}\right]$ is changed.

\section{DFT CALCULATIONS}

To cross check the plausibility of the interpretation in terms of the mentioned model DFT calculations were performed on GaAs using the FP-LAPW program WIEN97..$^{37}$

Former theoretical calculations with clamped ions ${ }^{6}$ predicted a change in the bond-charge density that should give an intensity variation for low-indexed reflections only-and with the opposite sign.

All calculations of crystal properties use the threedimensional periodicity of the crystal to handle the problem of an infinite number of atoms in the ideal solid. An external homogeneous electric field destroys the periodicity in at least one direction-and thus prohibits an exact treatment of this problem. ${ }^{3}$ Possible ways out are to calculate a finite slab or to introduce a periodic potential that is almost linear, but only in "interesting" regions. ${ }^{4,5}$ Here, we use a periodic sawlike potential $V_{\text {ext }}$ that is realized by the Fourier summation

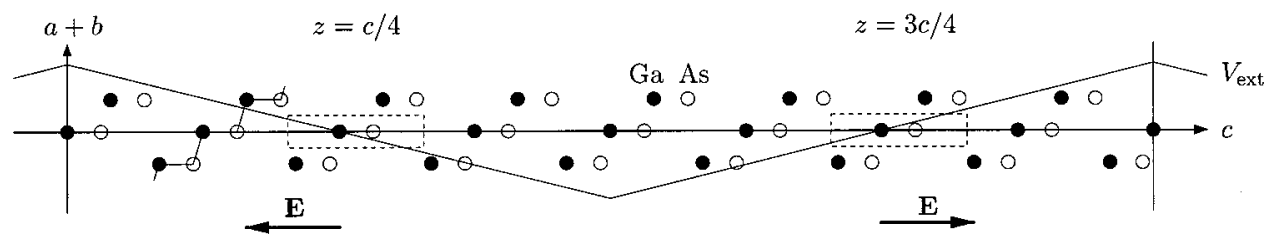

FIG. 7. Sketch of the cut through the super cell containing all 48 atoms. The $c$ axes is parallel to [111] of the cubic unit cell, the zigzag chains follow the direction [110] of the cubic cell. The dashed line represents the external potential. Within the super cell, both field directions are realized at the same time. The atoms at $c \approx 0.25$ and $c \approx 0.75$ are surrounded by eight coordination spheres, all in the homogeneous region of the external potential. The regions within the dotted boxes were used to calculate the theoretical structure factors.
FIG. 6. External potential $V_{\text {ext }}$ calculated according to Eq. (12) with $m=8$ (solid line). The dashed line displays the difference to the ideal $(m \rightarrow \infty)$ homogeneous potential, magnified by 100 .

$$
\begin{gathered}
V_{\text {ext }}=V_{0} \frac{8}{\pi^{2}} \sum_{n} \frac{1}{n^{2}} \cos \left[n z \frac{2 \pi}{c}\right] ; \\
n=1,3,5 \ldots 2 m-1,
\end{gathered}
$$

where $c$ is the period of $V_{\text {ext }}$ and $z$ is the absolute coordinate parallel to c. In Fig. $6, V_{\text {ext }} / V_{0}$ is displayed for $m=8$ together with the difference from the ideal $(m \rightarrow \infty)$ potential. A choice of $m>8$ leads to sharper kinks at $z=0$ and $z$ $=0.5 c$ but may cause problems in the Fourier transformation. Since $V_{\text {ext }}$ is small compared to the potential of the unperturbed crystal $V_{\text {int }}$, the wiggles in $V_{\text {ext }}$ caused by the truncation of the Fourier series can be neglected.

WIEN97 is based on the atomic spheres but goes beyond the "muffin tin" approach. This means that the crystal is subdivided into spherical nonoverlapping regions centered at the nuclei and the remaining "interstitial." In the interstitial $V_{\text {int }}$ is expanded in a Fourier series, $V_{i} \exp \left[i \mathbf{k}_{i} \mathbf{r}\right]$, fulfilling the lattice symmetry. To introduce $V_{\text {ext }}$ the Fourier coefficients $V_{i}$ with $\mathbf{k}_{i} \mathbf{r}=2 \pi n / c$ from Eq. (12) are increased by harmonics and $V_{\text {ext }}$ has to be added explicitly as given in Eq. (12). For this purpose, the WIEN97 code had to be modified accordingly.

The period $c$ of $V_{\text {ext }}$ determines the length of the super cell. For all calculations the cell was chosen to be hexagonal with the $c$ axis parallel to [111] of the cubic unit cell and $c$ $=8 \sqrt{3} a_{0}$ (see Fig. 1), i.e., it contains 24 units of GaAs, arranged as shown in Fig. 7.

For numerical reasons, the accuracy of the calculated potential $V_{\text {int }}$ is of the order of the maximum potential applied $V_{0} 8 / n^{2} \pi^{2}$. Inside the spheres $V_{\text {int }}$ is developed in spherical 
in the experiments, i.e., $1 \mathrm{mRy}$. (The unit $1 \mathrm{Ry}=13.6 \mathrm{eV}$ is used in WIEN97.) In order to obtain numerically reliable results in our computations, the external potentials were increased to $V_{0}=100 \mathrm{mRy}$ and $V_{0}=200 \mathrm{mRy}$, corresponding to field strengths of 700 and $1400 \mathrm{kV} \mathrm{mm}^{-1}$ - about 200 times larger than the experimental values.

For the integration in the $k$ space, 21 nonequivalent points were used, since a preliminary run with $7 k$ points showed inconsistencies in the charge density for $V_{0}=100 \mathrm{mRy}$.

A problem that has to be considered is that the width of the electronic band gap in GaAs is underestimated by DFT calculations, as is well known. In the present case, the band gap of GaAs was calculated to be $0.26 \mathrm{eV}$ instead of the experimental value $1.52 \mathrm{eV}(T=0 \mathrm{~K}) .{ }^{38} \mathrm{~A}$ further band-gap reduction caused by the external potential may result in "metallic GaAs" and lead to nonphysical charge densities. For that reason the existence of a band gap was checked for all self-consistent field calculations. The minimum band gap calculated with an external potential was $0.08 \mathrm{eV}$.

Also, the total charges in the spheres were checked to make sure that the super cell is large enough. There is a small charge accumulation at the kinks of the potential but no significant charge transfer, neither from one half cell to the other, nor between the cation and anion spheres.

In a first step, the positions of the nuclei were held fixed (clamped ion geometry, as used by Resta and Baldereschi ${ }^{6}$ ). The structure factors calculated for the entire super cell can not be used for a comparison with experimental data because they "contain" both field directions and the discontinuities at $z=0$ and $z=0.5 \mathrm{c}$. This problem can be overcome by using the contributions to $F$ from selected spheres only, values that are provided by WIEN97. The disadvantage of this procedure is that changes in the interstitial are not considered. To estimate the error introduced by neglecting the interstitial, $\Delta F_{222}$ was calculated by Fourier summation over a repeated section of the charge density (the dotted areas in Fig. 7). The modulus of $\Delta F_{222}$ derived this way is about $14 \%$ larger than by neglecting the interstitial. Nevertheless, the incomplete atomic contributions should reflect the essential electrondensity response to $V_{\text {ext }}$. Also, using these quasiatomic form factors and neglecting the interstitial has the advantage that experimental conditions like anomalous dispersion and room temperature can be added to the theoretical data by inserting the quasiatomic structure factors from the spheres as $f_{a}$ and $f_{c}$ in Eq. (5) (without $f_{b c}$ ). For the subsequent discussion, the structure factors used are calculated in this way.

The charge density calculated with clamped ions (Fig. 8) agrees, in the bond region, with the one calculated by Resta and Baldereschi with a pseudopotential approach. ${ }^{6}$ This difference density may be interpreted in terms of a shift of the bond charge relative to the nuclear positions against the external field direction, accompanied by an increase (decrease) of the bond charge.

As already discussed in Sec. IV the measured effect is essentially caused by the displacement of the nuclei. Thus, in the next step the positions of the nuclei were refined so that the resulting forces acting on them vanish. These forces (computed by WIEN97) are caused by the other nuclei, the electrons and the external potential. The force on one

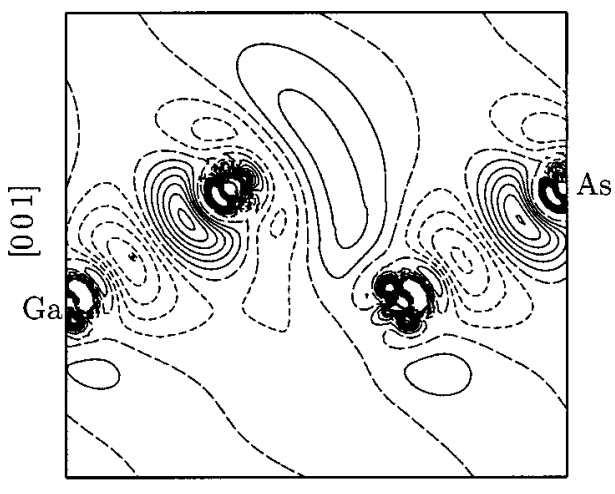

$[110]$

FIG. 8. Difference charge-density map for GaAs in the [1 $1 \overline{1} 0]$ plane through the origin of the unit cell. E E\|[ $[\overline{1} \overline{1}], \quad E$ $=1400 \mathrm{kV} \mathrm{mm}^{-1}$. Solid lines are positive, dashed lines are negative areas. The contour line distance is $2.5 \times 10^{-5} \mathrm{e} \mathrm{Bohr}^{-3}$.

nucleus caused by an external potential with $V_{0}=200 \mathrm{mRy}$ is of the order of $90 \mathrm{mRy} / \mathrm{Bohr}$. The corresponding variation of the bond length is about $0.02 \AA$. These values are in agreement with the experimentally determined dependence of the bond length upon the external electric-field strength.

The "relative intensity variations" calculated from the resulting structure factors with Eq. (5) are displayed in Fig. 9. The nonlinear dependence of $(\Delta R / R)$ on $E$ in the range $-1600 \mathrm{kV} \mathrm{mm}^{-1}<E<1600 \mathrm{kV} \mathrm{mm}^{-1}$ can be entirely explained by the change of the phase factor $\exp [i \mathbf{k}(\mathbf{r}+\mathbf{u})]$ for
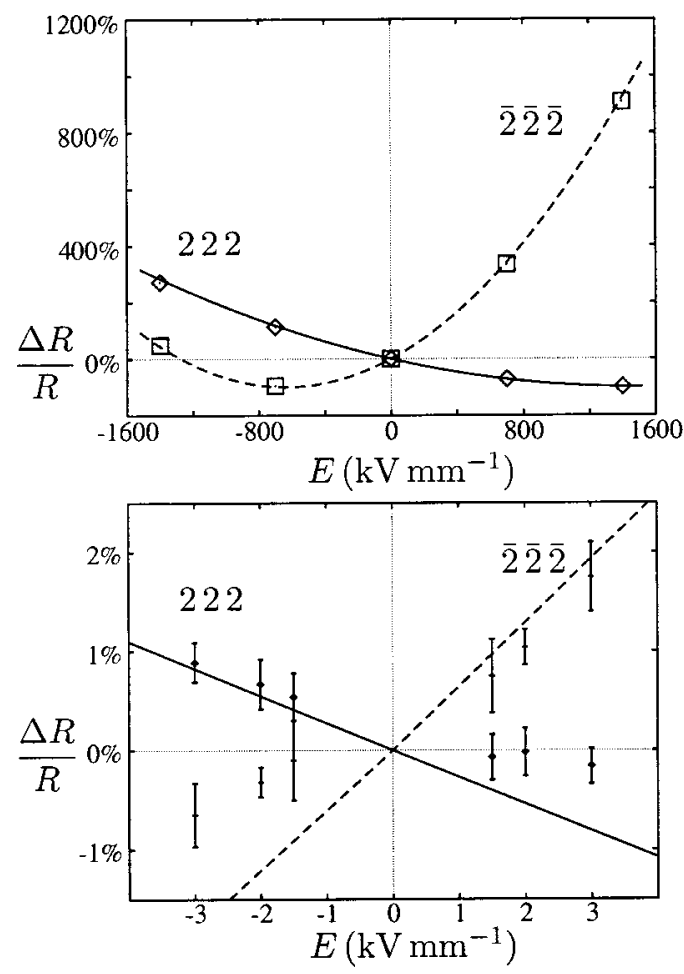

FIG. 9. Relative intensity variation of the GaAs reflections 222 and $\overline{2} \overline{2} \overline{2}$ calculated with WIEN97 and fitted curves (top). In the lower panel a close up of the region close to $E \approx 0$ is shown in comparison to the experimental values (see Fig. 2). 
large values of $\mathbf{u}$. The interpolation of the calculated values down to the range $-3 \mathrm{kV} \mathrm{mm}^{-1}<E<3 \mathrm{kV} \mathrm{mm}^{-1}$ does not show any nonlinear behavior.

The calculated values for $(\Delta R / R)$ in the range $-3 \mathrm{kV} \mathrm{mm}{ }^{-1}<E<3 \mathrm{kV} \mathrm{mm}^{-1}$ are of the order of the experimentally determined ones for one field direction and reproduce the outcome of the semiempirical model (see Table I). Taking into account both the assumptions made in the calculation and in the data reduction, this is a remarkable result. Changes in the electron density in the bond region and a polarization near the nuclei play a minor role compared to the displacement of the atomic cores.

The difference-density $\rho_{E}-\widetilde{\rho}_{0}$, where $\widetilde{\rho}_{0}$ is the electron density calculated for displaced ions but with $V_{\text {ext }}=0$, agrees qualitatively and quantitatively with $\rho_{E}-\rho_{0}$ as shown in Fig. 8. The charge-density response in the bond region is over compensated by the increased (decreased) overlap of the atomic charge clouds resulting from the displacement of the nuclei. It does not make much sense to calculate a difference-density $\rho_{E}-\rho_{0}$ with displaced ions because the choice of the common origin is arbitrary.

The comparison of experimental and calculated relative intensity variations suffers from the following shortcomings: For numerical reasons $V_{\text {ext }}$ had to be 2 orders of magnitude larger than the experimental potentials. To observe a response in the charge density to a small field of $V_{\text {ext }}$ $=1 \mathrm{mRy}$ is beyond the computational accuracy. The distortion of the unit cell (the macroscopic inverse piezoelectric effect) was neglected. Although this distortion hardly affects the measured quantities, it leads to a variation of the bond lengths and, thus, to changed forces on the nuclei. The adaption of the theoretical data to the experimental conditions by Eq. (5) is only a crude approximation. ${ }^{7}$ The error introduced this way can be estimated by comparing the order of the measured and the calculated effect in the lower part of Fig. 9.
On the other hand, there is no model-free way to reduce the experimentally determined $(\Delta R / R)$ to $\Delta F$ at $T=0 \mathrm{~K}, \lambda$ $=0 \AA$.

\section{CONCLUSION}

Both, the experimental determination of the relative intensity variations of $x$-ray reflections caused by an external electric field and its theoretical calculation represent the state of the art.

The experimental data must be interpreted in terms of a semiempirical model that represents the physical hypothesis as close as possible. At the same time, the model has to consider the experimental conditions (room temperature, anomalous dispersion, ...). For ab initio calculations, the situation is slightly different. They do not depend on a model to calculate measurable quantities as long as the experimental constraints can be included in the calculation. So far this is not possible for $T \gg 0$, anomalous dispersion, and an homogeneous electric field, so that the DFT calculation describes a system that is different from the experimentally probed one and one has to use a model again to compare both.

Keeping this in mind, theory and experiment show qualitative and quantitative agreement (except for the different slopes for opposite field directions in the experimental data). This allows us to explain the measured effect by a small displacement of the cation and anion sublattices relative to each other.

\section{ACKNOWLEDGMENTS}

The authors thank H.-G. Krane, W. Morgenroth, and A. Berghäuser for experimental assistance at HASYLAB. This project was supported by the EU (CHRX-CT-93-0155) and by the BMBF (05 647IPA).
*Electronic address: upietsch@rz.uni-potsdam.de

${ }^{1}$ R. M. Martin and G. Ortiz, Solid State Commun. 102, 121 (1997).

${ }^{2}$ R. D. King-Smith and D. Vanderbilt, Phys. Rev. B 47, 1651 (1993).

${ }^{3}$ R. Resta, Rev. Mod. Phys. 66, 899 (1994).

${ }^{4}$ K. Kunc and R. Resta, Phys. Rev. Lett. 51, 686 (1983).

${ }^{5}$ R. Resta and K. Kunc, Phys. Rev. B 34, 7146 (1986).

${ }^{6}$ R. Resta and A. Baldereschi, Phys. Rev. B 23, 6615 (1981).

${ }^{7}$ J. Stahn, M. Möhle, and U. Pietsch, Acta Crystallogr., Sect. B: Struct. Sci. 54, 231 (1998).

${ }^{8}$ S. Haussühl, Kristallphysik (VEB Deutscher Verlag für Grundstoffindustrie, Leipzig, 1983).

${ }^{9}$ C. S. G. Cousins, Acta Crystallogr., Sect. A: Found. Crystallogr. 39, 257 (1983).

${ }^{10}$ C. S. G. Cousins, J. Phys. C 15, 1857 (1982).

${ }^{11}$ C. S. G. Cousins, L. Gerwald, K. Nielsen, J. Staun Olsen, B. Selsmark, B. J. Sheldon, and G. E. Webster, J. Phys. C 15, L651 (1982).

${ }^{12}$ C. S. G. Cousins, L. Gerward, J. Staun Olsen, B. Selsmark, and B. J. Sheldon, J. Appl. Crystallogr. 15, 154 (1982).

${ }^{13}$ C. S. G. Cousins, J. Appl. Crystallogr. 21, 496 (1988).

${ }^{14}$ C. S. G. Cousins, J. Phys. C 14, 4553 (1981).
${ }^{15}$ I. Fujimoto, Jpn. J. Appl. Phys. 19, L345 (1980).

${ }^{16}$ U. Pietsch, J. Mahlberg, and K. Unger, Phys. Status Solidi B 131, 67 (1985).

${ }^{17}$ J. Stahn, A. Pucher, T. Geue, A. Daniel, and U. Pietsch, Europhys. Lett. 44, 714 (1998).

${ }^{18}$ J. Stahn, Ph.D. thesis, Universität Potsdam, 1999.

${ }^{19}$ J. B. Mullin, B. W. Staughan, C. M. H. Driscoll, and A. F. W. Willoughby, Inst. Phys. Conf. Ser. 24, 275 (1975).

${ }^{20}$ B. J. Skinner and P. B. Barton, Jr., Am. Mineral. 45, 2261 (1960).

${ }^{21}$ G. Arlt and P. Quadflieg, Phys. Status Solidi 25, 323 (1968).

${ }^{22}$ D. Berlincourt, H. Jaffe, and L. R. Shiozowa, Phys. Rev. 129, 1009 (1963).

${ }^{23}$ D. L. Rode, in Semiconductors and Semimetals, edited by W. R. K. and A. C. Beer (Academic, New York, 1975).

${ }^{24}$ Landolt and Börnstein, New Series, Numerical Data and Functional Relationships, in Science and Technology, edited by $\mathrm{O}$. Madelung, Group III, Vol. 17 (Springer-Verlag, Berlin, 1982).

${ }^{25}$ M. Möhle, Ph.D. thesis, Universität Potsdam, 1997.

${ }^{26}$ G. J. McIntyre, G. Moss, and Z. Barnea, Acta Crystallogr., Sect. A: Cryst. Phys., Diffr., Theor. Gen. Crystallogr. 36, 482 (1980).

${ }^{27}$ U. Pietsch, K. Paschke, and K. Eichhorn, Acta Crystallogr., Sect. B: Struct. Sci. 49, 822 (1993). 
${ }^{28}$ A. Hadni, J. Claudel, and P. Strimer, Phys. Status Solidi 26, 241 (1968).

${ }^{29}$ J. A. Bearden and A. F. Burr, Rev. Mod. Phys. 39, 125 (1967).

${ }^{30}$ J. C. Phillips, Phys. Rev. 166, 832 (1968).

${ }^{31}$ J. A. van Vechten, Phys. Rev. B 10, 4222 (1974).

${ }^{32}$ Z. G. Pinsker, Dynamical Scattering of X-Rays in Crystals (Springer, Berlin, 1978).

${ }^{33}$ E. Weckert and K. Hümmer, Acta Crystallogr., Sect. A: Found. Crystallogr. 53, 108 (1997).

${ }^{34}$ J. Stahn, A. Pucher, U. Pietsch, J. Zellner, and E. Weckert, Acta Crystallogr., Sect. A: Found. Crystallogr. 55, 1034 (1999).
${ }^{35}$ B. T. M. Willis, Acta Crystallogr., Sect. A: Cryst. Phys., Diffr., Theor. Gen. Crystallogr. 25, 277 (1969).

${ }^{36}$ R. Dovesi, V. R. Saunders, C. Roetti, M. Causa, N. M. Harrison, R. Orlando, and E. Aprà, CRYSTAL95, User's Manual (Theoretical Chemistry Group-University of Turino, CCLRC Daresbury Laboratory, UK, 1995).

${ }^{37}$ P. Blaha, K. Schwarz, and J. Luitz, WIEN97, A Full Potential LAPW Package for Calculating Crystal Properties (K. Schwarz, TU Vienna, Austria, 1999), ISBN Report No. 3-9501031-0-4 (unpublished).

${ }^{38}$ B. J. Skromme and G. E. Stillman, Phys. Rev. B 29, 1982 (1984). 